\title{
Active vibration control of periodic disturbance based on the lattice notch filter
}

\author{
Jinchun Liu ${ }^{1, a^{*}}$,Jingjun Lou ${ }^{1, a}$, Qiwei He ${ }^{1, b}$ and Zhang Zhen ${ }^{1, c}$ \\ ${ }^{1}$ Naval University of Engineering, China \\ aliu2015_liu@163.com, bhe_1980_he@sina.com, 'hangzhen_1992@tom.com
}

\begin{abstract}
Keywords: Lattice notch filter; active control; vibration control.
\end{abstract}
Abstract. A narrowband active vibration control algorithm with frequency estimator based on lattice notch filter is proposed to suppress the primary signal with multi line spectra. A adaptive iir (infinite impulse response) lattice gradient notch filter was adapted to estimated the frequency of the multi-harmonics buried in the primary signal, then the reference signal is filtered by the adaptive band pass filter. With the reference signal filtered by the adaptive band pass filter, active control algorithm is implemented. Simulations of the frequency estimation of the multi-sinusoidal signal are conducted and simulation results confirm that the proposed algorithm could achieve effective performance when the frequency of the primary disturbance is unknown.

\section{Introduction}

Active control of vibration has finds a lot application such as fans, engines, and air conditioners in nowadays. The basic concept of active vibration control is to suppress the primary disturbance with the secondary force which is of same amplitude and opposite phase. Signals generated by the rotating machines such as fans, diesel engines, and air conditioners is characterized with discreet frequency which cause damage to operator in the working environment. Narrowband active vibration control (NAVC) is an effective method to suppress such vibration with multi-line spectra.

The application of NAVC has been implemented taken into applications since 1970's. A lot of algorithms have been proposed for suppress the primary disturbance with multi-spectral. There exists two families of algorithm in AVC system, which composed of filtered-X least mean square (FXLMS) algorithm and filtered-X recursive least square algorithm (FXRLS). Due to its simplicity and implementation efficiency, FXLMS algorithm is the most popular adaptive algorithm to suppress the primary disturbance compared to FXRLS algorithms. In the application of NAVC systems, the performance of NAVC systems is directly correlated with the reference signal and the primary signal. NAVC algorithm could not achieve effective performance if the correlation between the primary signal and the reference signal is weak. Frequency estimation of the primary signal is an effective method to adapt to improve the relativity between the reference signal and the primary signal. To estimate the frequency of a sinusoidal signal, a number of algorithm has been proposed to exploit the predictable nature of sinusoidal primary disturbance and characterize the frequency of the sinusoidal primary disturbance, such the Kalman algorithm, music, FFT (fast Fourier transform), etc. such method could be capable of tracking nonstationary or slowly-varying primary signals. However, they have some shortcomings in the application of NAVC systems. a lot of Kalman-based algorithms have been proposed for the frequency estimation of the sinusoidal signal in many applications such as the electrical power system, however, it requires much more computation burdens which is much more complicated compared the other algorithms. FFT methods have been proposed in the past to estimated the frequency of the primary signal, but it is has been found that the estimated result could not be accessed effectively in real-time of AVC system. Adaptive notch filter has been proposed for the frequency estimation of the sinusoidal signal in a lot of applications. Adaptive lattice filter shows much more stability and performance compared to the bilinear adaptive notch filter. In this paper, the adaptive lattice notch filter is introduced into an adaptive vibration control system to estimate the frequency of synthesized desired signal. Then, the reference signal is generated to suppress the primary vibration in the active vibration control system. 


\section{Frequency Estimator Based on Lattice Filter}

The reference signal generator relies on a method of the frequency estimator based on the adaptive notch filter. In this section, an adaptive lattice notch filter is adapted to estimated the frequency of the synthersized primary signal. A second order iir lattice notch filter depicted is given as follows

$$
H(z)=\frac{1+\sin \theta_{2}}{2} \frac{1+2 \sin \theta_{1} z+z^{2}}{1+\sin \theta_{1}\left(1+\sin \theta_{2}\right) z+z^{2}}
$$

inwhich $\theta_{1}$ is the parameter which has a relationship about the notch frequency $\omega_{0}$ which is expressed as $\omega_{0}=\theta_{1}+\frac{\pi}{2}$. The relationship between the bandwidth and the parameter $\theta_{2}$ is $\sin \theta_{2}=\frac{1-\tan (B / 2)}{1+\tan (B / 2)}$. Therefore, the parameter of bandwidth $B$ is unrelated with $\theta_{1}$, and the notch frequency is determined by $\omega_{0}$. The adaptive algorithm for the parameter $\theta_{1}(n)$ is given as follow

$$
\theta_{1}(n+1)=\theta_{1}(n)-\mu y(n) x(n) \hat{\omega}(n)
$$

inwhich $\mu$ is the step $y(n)$ is the output of the lattice filter and $x(n)$ is given by

$$
x(n)=\frac{z \cos \theta_{1} \cos \theta_{2} u(n)}{1+\sin \theta_{1}\left(1+\sin \theta_{2}\right)+z^{2}}
$$

Multi-frequency case: cascaded structure

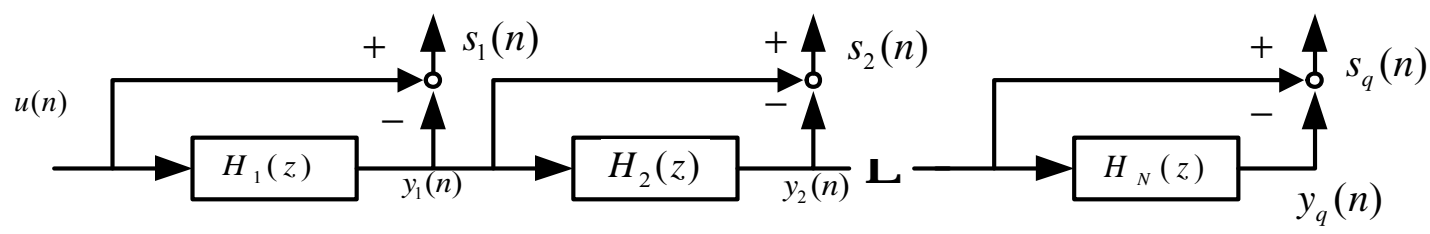

Fig. 1 cascaded adaptive notch filter

In order to identify the multi-frequecy of multi-sinusoidal signals buried in the backgroud noise, the single frequency case is extended in to the multi-frequency case with the cascaded structure as depicted in Fig.1. $u(n)$ is the input signal of cascaded adaptive notch filter which is composed of $N$ sinusoidal signal. The output of the $i$ th lattice notch filter is $y_{i}(n), s_{i}(n)$ is the $i$ th filtered sinusoidal signal.

The relationship between the output $N$ th signal $y_{N}(n)$ and the input signal $u(n)$ is shown as follows

$$
\frac{y_{N}(z)}{u(z)}=\prod_{i=1}^{N} H_{i}(z)
$$

where $y_{i}(z)$ and $u(z)$ is the Z-transformation of signl $y_{i}(n), u(n)$ respectively. $H_{i}(z)$ is the $i$ th transferr function for the $i$ th sinusoidal.

\section{Adaptive Feedback AVC algorithm based on frequency estimator}

The proposed algorithm is composed of three parts. Firstly, the frequency of the synthesized primary signal is estimated. Generally, the exact frequency of the primary vibration is not easy to be obtained. But, the initial value of estimated frequency could be could be accessed and set to the rated value of the rotating machinery. Because, although, the primary vibration generated by rotating machinery which exact rotational speed is not identical to rated value which is caused by the supplied voltage and the conditions. 
Secondary, the reference signal is generated by the sinusoidal signal generator as

$$
X(n)=\left[x_{1}(n) \mathrm{L} x_{q}(n)\right]^{T}, x_{i}(n)=\left[\cos \hat{\omega}_{i} n \sin \hat{\omega}_{i} n\right]^{T}, i=1,2, \ldots q
$$

where $q$ is the number of the estimated frequency of the synthesized primary vibration signal. $x_{i}(n)$ is the sine and cosine sinusoidal signal generated by the $i$ th estimated frequency.

Thirdly, the completely parallel form of adaptive feedback AVC system is implemented.

$$
\hat{p}(n)=\hat{s}(n) *\left[W^{T}(n) X(n)\right]+e(n), W(n)=\left[h_{1,0}, h_{1,1} \ldots h_{q, 0}, h_{q, 1}\right]^{T}
$$

where $\mathrm{W}(n)$ is the adaptive controller filter, $\hat{s}(n)$ is the estimated secondary path, $*$ denotes a convolution operator. The synthesized primary signal $\hat{p}(n)$ is the estimated value of the primary vibration $p(n)$ generated by the rotating machinery. The primary vibration is composed of the harmonic signal and the background vibration $v(n)$ with variance of $\sigma^{2}$.

$$
p(n)=\sum_{i=1}^{q} A_{i} \cos \left(\omega_{i} n+\phi_{i}\right)+v(n)
$$

where $\omega_{i}$ is the $i$ th harmonic signal of the primary signal. $\phi_{i}$ is the intial phase angle of the $i$ th harmonic signal, $A_{i}$ is the amplitude of the corresponding harmonic signal. They would be identical when the estimated secondary path is perfect. the adaptive control filter is adapted as

$$
\begin{aligned}
& W(n+1)=W(n)+2 \mu e(n) X^{\prime}(n) \\
& X^{\prime}(n)=\left[x_{1}^{\prime}(n) \mathrm{L} \quad x_{q}^{\prime}(n)\right]^{T}, x_{i}^{\prime}(n)=\hat{s}(n) * x_{i}(n), i=1,2, \ldots q
\end{aligned}
$$

where $x_{i}^{\prime}(n)$ is the $i$ th filtered reference signal. Besides, the residual error signal $e(n)$ is expressed as

$$
e(n)=d(n)-s(n) *\left[W^{T}(n) X(n)\right]
$$

where $s(n)$ is the secondary path.

\section{Simulation Results}

The proposed AVC system for the single and mult-sinusoidal signal case is conducted in the simulations. Firstly, single frequency case was conducted. The normalized frequency of the primary signal is $0.10 \pi$. The signal vibration ratio is $20 \mathrm{~dB}$. The bandwidth parameter $\theta_{2}$ is set to $0.3 \pi$. Step size $\mu$ in the frequency recursion is set to $0.005, \mu_{w}$ is set to 0.05 . The initial estimated frequency of the reference signal was the prior knowledge which was set to $\hat{\omega}_{1}(1)=0.5 \pi$. It is clear that in Fig. 2(a) the frequency of reference frequency could converge to the frequency of the primary signal. Besides, the primary disturbance was mitigated as depicted in Fig. 2(b).

Secondly, Multi-frequency case was conducted. The normalized frequency of the primary signal is $0.01 \pi$ and $0.20 \pi$. The signal vibration ratio is $30 \mathrm{~dB}$. The initial frequency of the reference signal was the prior knowledge which was set to $\hat{\omega}_{1}(1)=0.05 \pi$ and $\hat{\omega}_{2}(1)=0.09 \pi$. The other parameter is the same as in single frequency case. It is depicted in Fig. 3that 


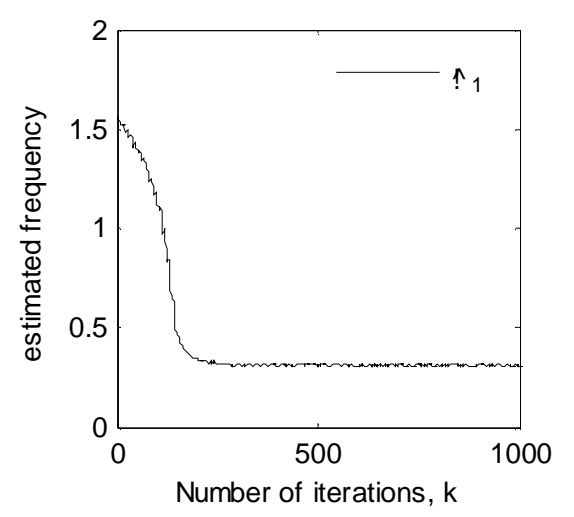

(a) estimated frequency $\hat{\omega}_{1}(n)$

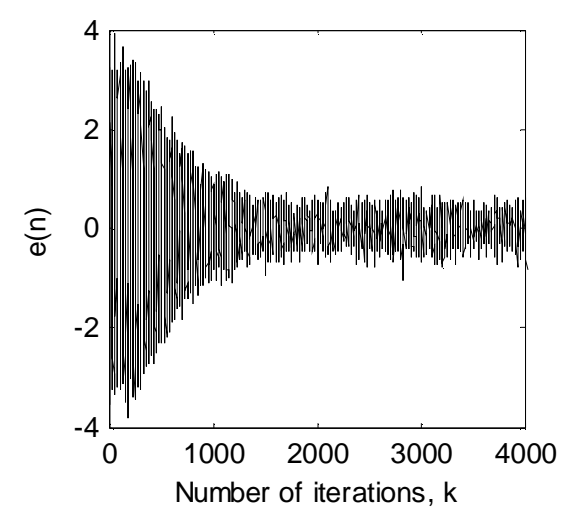

(b) residual error signal $e(n)$

Fig. 2 Performance of the proposed algorithm: single frequency case

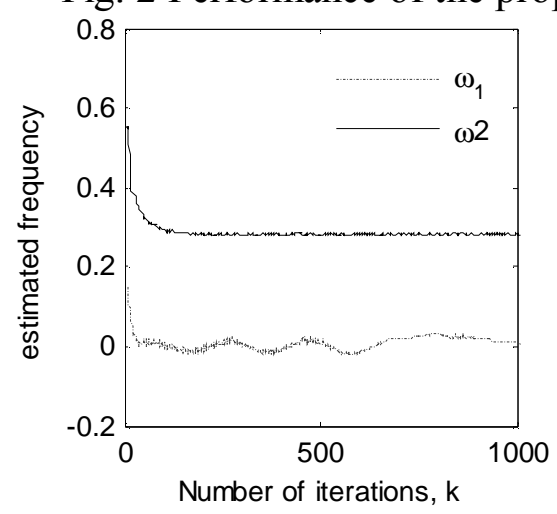

(a) estimated frequency $\hat{\omega}_{1}(n)$ and $\hat{\omega}_{2}(n)$

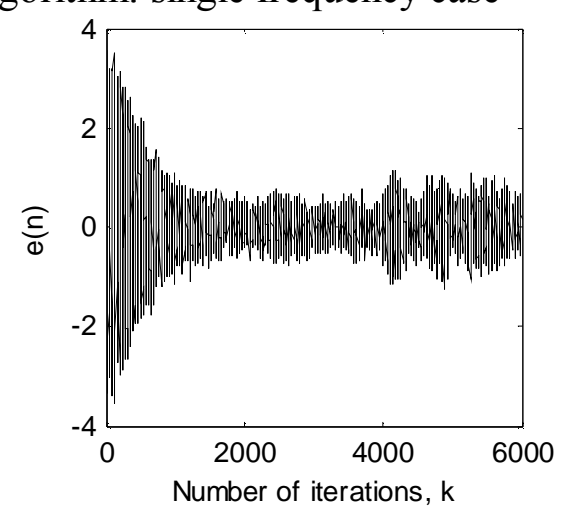

(b) residual error signal $e(n)$

Fig. 3 Performance of the proposed algorithm: multi-frequency case

\section{Summary}

When the frequency of the reference signal is not consistent with the frequency of primary signal, the performance of Narrowband AVC algorithm will degrades. To tackle this problem, an improved algorithm based on adaptive feedback AVC algorithm was proposed. In the proposed algorithm, cascade structure of the adaptive lattice notch filter was taken as the frequency estimtor to estimate the frequency of the primary distturbnce signal. The validity of the proposed algorithm for correcting the frequency error betweeen the reference singal and the primary signal in AVC system was confirmed in simluations.

\section{References}

[1] Elliott, S.J., Signal processing for active control, Academic press, 2001.

[2] Inman, D.J. and J.C. Simonis, Vibration control and active vibration suppression. New York, N.Y, 1987.

[3] Hansen, C.H., Active control of vibration and vibration., Boca Raton, FL: Taylor \& Francis, 2013.

[4] Kuo, S.M., D.R. Morgan, Active vibration control systems :algorithms and DSP implementations. New York: Wiley, 1995.

[5] KIM, S., P.Y., on-line fundamental frequency tracking method for harmonic signal and application to anc. Journal of Sound and Vibration, 241(4): pp. 681 - 691. 2001.

[6] KIM, S., P.Y., active control of multi-tonal vibration with reference generator on on-line frequency estimation. Journal of Sound and Vibration, 227(3): pp. 647 - 666. 1999. 
[7] Yazdanian, M., M. Mojiri ,F. Sheikholeslam. An extended Kalman filter for identification of biased sinusoidal signals. in Electrical Engineering (ICEE), 2012 20th Iranian Conference on. 2012.

[8] A. Routray, A. K. Pradhan, K. P. Rao, A novel Kalman filter for frequency estimation of distorted signals in power systems," IEEE Trans. Instrum. Measur. , vol. 51, no. 3, pp. 469-479, 2002.

[9] P. K. Dash, A. K. Pradhan, G. Panda, "Frequency estimation of distorted power system signals using extended complex Kalman filter," IEEE Trans. Power Del. , vol. 14, no. 3, pp. 761-766, 1999.

[10] Yegui, X., Shigeyasu, Tetsuya, Matsuno, et al., A New Robust Narrowband Active Vibration Control System in the Presence of Frequency Mismatch. Audio, Speech, and Language Processing, IEEE Transactions on, 14(6): pp. 2189-2200, 2006.

[11]A. V. Oppenheim, R. W. Schafer, and J. R. Buck, Discrete-Time Signal Processing, 2nd ed. Upper Saddle River, NJ: Prentice-Hall, 1998.

[12]Regalia, P.A., Adaptive IIR filtering in signal processing and control. Electrical engineering and electronics. New York: M. Dekker, 1995. 